\title{
Bayesian TV Denoising of SAR images
}

\author{
Miguel Vega ${ }^{(a)}$, Javier Mateos ${ }^{(b)}$, Rafael Molina ${ }^{(b)}, \operatorname{Aggelos~K.~Katsaggelos~}^{(c)}$ \\ (a) Dept. de Lenguajes y Sistemas \\ Informáticos \\ Univ. de Granada \\ 18071 Granada, Spain \\ Email: mvega@ugr.es \\ (c) Dept. of Electrical Engineering
and Computer Science
Northwestern Univ.
Evanston, Illinois 60208-3118
Email: aggk@eecs.northwestern.edu
}

\begin{abstract}
Synthetic aperture radar (SAR) imagery suffers from the speckle phenomenon. Speckle gives rise to the presence of multiplicative noise which severely degrades the observed images. It is known that logarithmically transformed speckle can be well approximated by a Gaussian distribution. In this paper we propose an algorithm for despeckling images, within the log-transformed spatial domain, using a TV prior whose model parameter is automatically determined using the Evidence Analysis within the Hierarchical Bayesian Paradigm. The effectiveness of the proposed algorithm, over both synthetically speckled and real SAR images, is studied.

Index Terms-SAR images denoising, despeckling, parameter estima-
\end{abstract} tion, Bayesian methods, image restoration.

\section{INTRODUCTION}

Synthetic aperture radar (SAR) satellite and airborne sensors gather a huge amount of all time, all weather, valuable information. It is well known (see [1]) that SAR, as other coherent imagery, like ultrasound and laser scans, suffer from the speckle phenomenon. Speckle is due to the interferences of waves reflected by many elementary reflectors inside a resolution cell, which, when fully developed, give rise to the presence of multiplicative noise. Fully developed speckle appears when the scanned surface is extremely rough compared to the beam wavelength. In this paper we only consider fully developed speckle. Speckle seriously damages the usability of observed images. A way to mitigate speckle is to take many samples per resolution cell, which is known as multi-look, but at the cost of a loss of resolution.

Many approaches to the problem of denoising speckled images, also known as despeckling, have been proposed. Median and adaptive filters, based on local statistics like the method in [2], have been applied (see [3]). The problem has also been studied within the wavelet and other transformed domains (see [4]-[6]), and several Total Variation (TV) applications to despeckling have appeared (see [6]-[9]). Some methods work directly with the observed images [2], [3], [7], [9], while others first apply a log-transform to the images, in order to convert multiplicative into additive noise [4]-[6], [8].

In this paper we propose a simple algorithm for despeckling images, within the log-trasformed spatial domain, using a TV prior. The model parameter is automatically determined using the Evidence Analysis within the Hierarchical Bayesian Paradigm [10].

The rest of this paper is organized as follows. Section II provides the mathematical model for speckled images acquisition process. We provide the description of the hierarchical Bayesian framework modeling the unknowns in Section III. The inference procedure utilized to develop the proposed method is presented in Section IV. We demonstrate the effectiveness of the proposed approach with

This work was supported in part by the Comisión Nacional de Ciencia y Tecnología under contract TIN2010-15137 and the Spanish research programme Consolider Ingenio 2010: MIPRCV (CSD2007-00018). experimental results in Section V. Finally, section VI concludes the paper.

\section{Problem Formulation}

The degradation model for an $L$-looks observed image $\boldsymbol{g}$, of size $p=s \times t$, with elements $g_{i}$ subject to fully developped speckle noise, from the noise free original image $f$, of the same size, with elements $f_{i}$, is the following

$$
g_{i}=f_{i} \mu_{i}, \text { for } i=1, . ., p .
$$

Therefore $\boldsymbol{\mu}$, of size $p$, with elements $\mu_{i}$, in Eq. (1), follows the Gamma distribution

$$
\mathrm{p}\left(\mu_{i}\right)=\Gamma\left(\mu_{i} \mid L, L\right), \text { for } i=1, . ., p,
$$

with

$$
\Gamma(\omega \mid a, b)=\frac{b^{a}}{\Gamma(a)} \omega^{a-1} \exp [-b \omega],
$$

for $\omega>0$, where $a>0$ and $b>0$ are the shape and inverse scale parameters, respectively. Its mean and variance are $\mathrm{E}[\omega]=a / b$, $\operatorname{Var}[\omega]=a / b^{2}$. So, for $\mathrm{p}\left(\mu_{i}\right)$ in Eq. (1) we have $\mathrm{E}\left[\mu_{i}\right]=1$ and $\operatorname{Var}\left[\mu_{i}\right]=\frac{1}{L}$.

It has been shown (see [11]) that logarithmically transformed speckle noise approximately follows the Gaussian distribution, that is,

$$
\ln \mu_{i} \sim \mathcal{N}\left(\mu_{i} \mid m, \sigma^{2}\right)
$$

with mean and variance

$$
m=\psi(L)-\ln L, \quad \sigma^{2}=\psi^{(1)}(L),
$$

where $\psi(z)=\frac{d}{d z} \ln \Gamma(z)$ is the digamma function, and $\psi^{(1)}(z)=$ $\frac{d}{d z} \psi(z)$ is the polygamma function of order 1 .

Let us define the logarithmically transformed images, of size $p$,

$$
y_{i}=\ln g_{i}
$$

and

$$
x_{i}=\ln \left(f_{i}\right)+m, \text { for } i=1, . ., p,
$$

where $m$ has been defined in Eq. (5). From Eqs. (1) and (4), we obtain the following degradation model, for the 'observed' $y$ from the unknown $\boldsymbol{x}$,

$$
\boldsymbol{y}=\boldsymbol{x}+\boldsymbol{\nu},
$$

with $\boldsymbol{y}, \boldsymbol{x}$ and $\boldsymbol{\nu}$, lexicographically arranged vectors of size $p, \boldsymbol{\nu} \sim$ $\mathcal{N}\left(\boldsymbol{\nu} \mid \mathbf{0}, \sigma^{2} \mathbf{I}\right), \sigma^{2}$ defined in Eq. (5), and $\mathbf{I}$ the $p \times p$ identity matrix.

Then to obtain an estimate of $\boldsymbol{f}$ from one of $\boldsymbol{x}$ one simply uses

$$
\hat{f}_{i}=\exp \left(\hat{x}_{i}-m\right), \text { for } i=1, \ldots, p,
$$


where $\hat{x}_{i}$ is an estimate of $x_{i}$ in Eq. (8) which is obtained with a denoising method (see [12]).

In this paper the estimate of $\hat{\boldsymbol{x}}$ is obtained by utilizing a TV prior on $\boldsymbol{x}$. The unknown parameter of this prior distribution is estimated by making use of the Evidence Analysis within the Bayesian framework. Chambolle's algorithm [13] is used to find the maximum ' $a$ posteriori' estimate of $\boldsymbol{x}$ for a TV prior.

\section{BAYESIAN MODELING}

Our problem is now stated in terms of a Bayesian inference about the unknown $\boldsymbol{x}$ from the transformed observed image $\boldsymbol{y}$. We will solve for $\boldsymbol{x}$ within the Hierarchical Bayesian Paradigm (see [10]).

In the hierarchical approach to image restoration we have at least two stages. In the first stage, knowledge about the structural form of the noise and the structural behavior of the restoration is used in forming $\mathrm{p}(\boldsymbol{y} \mid \boldsymbol{x})$ and $\mathrm{p}(\boldsymbol{x} \mid \alpha)$, respectively.

Given the degradation model defined in Eq. (8), the probability distribution $\mathrm{p}(\boldsymbol{y} \mid \boldsymbol{x})$ will be

$$
\mathrm{p}(\boldsymbol{y} \mid \boldsymbol{x})=\mathcal{N}\left(\boldsymbol{y} \mid \boldsymbol{x}, \sigma^{2} \mathbf{I}\right),
$$

with $\sigma^{2}$ known and defined in Eq. (5).

We will use a TV image prior [14], that is,

$$
\mathrm{p}(\boldsymbol{x} \mid \alpha) \propto \alpha^{\frac{p}{2}} \exp (-\alpha \mathrm{TV}(\boldsymbol{x}))
$$

with the model parameter $\alpha$, and

$$
\operatorname{TV}(\boldsymbol{x})=\sum_{i=1}^{p} \sqrt{\left(\Delta_{i}^{h}(\boldsymbol{x})\right)^{2}+\left(\Delta_{i}^{v}(\boldsymbol{x})\right)^{2}},
$$

where $\Delta_{i}^{h}(\boldsymbol{x})$ and $\Delta_{i}^{v}(\boldsymbol{x})$ are the horizontal and vertical first order differences of $\boldsymbol{x}$.

In the second stage, of the hierarchical approach, we incorporate knowledge about the model parameter $\alpha$, using the Gamma hyperprior, that is,

$$
\mathrm{p}(\alpha)=\Gamma\left(\alpha \mid a_{\alpha}^{o}, b_{\alpha}^{o}\right),
$$

where the Gamma distribution has been defined in Eq. (3).

Finally, the joint probability distribution of our model is

$$
\mathrm{p}(\boldsymbol{x}, \boldsymbol{y}, \alpha)=\mathrm{p}(\boldsymbol{y} \mid \boldsymbol{x}) \mathrm{p}(\boldsymbol{x} \mid \alpha) \mathrm{p}(\alpha),
$$

where the distributions $\mathrm{p}(\boldsymbol{y} \mid \boldsymbol{x}), \mathrm{p}(\boldsymbol{x} \mid \alpha)$ and $\mathrm{p}(\alpha)$ have been defined in Eqs. (10), (11), and (13), respectively.

\section{BAYESIAN INFERENCE}

Bayesian inference will be performed in the so-called Evidence Analysis approach [10]. In this approach $\hat{\alpha}$ is first selected as

$$
\begin{aligned}
\hat{\alpha} & =\underset{\alpha}{\operatorname{argmax}} \int \mathrm{p}(\alpha, \boldsymbol{x} \mid \boldsymbol{y}) \mathrm{d} \boldsymbol{x}=\underset{\alpha}{\operatorname{argmax}} \int \mathrm{p}(\alpha, \boldsymbol{x}, \boldsymbol{y}) \mathrm{d} \boldsymbol{x} \\
& =\underset{\alpha}{\operatorname{argmax}}\left\{\mathrm{p}(\alpha) \int \mathrm{p}(\boldsymbol{y} \mid \boldsymbol{x}) \mathrm{p}(\boldsymbol{x} \mid \alpha) \mathrm{d} \boldsymbol{x}\right\},
\end{aligned}
$$

and then $\hat{\boldsymbol{x}}$ as

$$
\hat{\boldsymbol{x}}=\underset{\boldsymbol{x}}{\operatorname{argmax}}\{\mathrm{p}(\boldsymbol{y} \mid \boldsymbol{x}) \mathrm{p}(\boldsymbol{x} \mid \hat{\alpha})\}
$$

Utilizing

$$
\mathcal{E}(\boldsymbol{x}, \alpha, \boldsymbol{y})=\left\{\frac{1}{2 \sigma^{2}}\|\boldsymbol{x}-\boldsymbol{y}\|^{2}+\alpha \operatorname{TV}(\boldsymbol{x})\right\},
$$

we have

$$
\hat{\alpha}=\underset{\alpha}{\operatorname{argmax}}\left\{\alpha^{\frac{p}{2}} \mathrm{p}(\alpha) \int \exp \{-\mathcal{E}(\boldsymbol{x}, \alpha, \boldsymbol{y})\} \mathrm{d} \boldsymbol{x}\right\}
$$

and

$$
\hat{\boldsymbol{x}}=\underset{\boldsymbol{x}}{\operatorname{argmin}} \mathcal{E}(\boldsymbol{x}, \hat{\alpha}, \boldsymbol{y}) .
$$

Unfortunately, we can not directly tackle the minimization in Eq. (18) because of the TV image prior. In earlier work with TV priors (see [15]), this difficulty is overcome by resorting to majorizationminimization (MM) approaches, which is also the method adopted in this paper.

The main principle of the MM approach is to find a bound of $\mathcal{E}(\boldsymbol{x}, \alpha, \boldsymbol{y})$ which makes the maximization of (18) tractable. Let us first consider the functional

$$
\mathrm{M}(\boldsymbol{x}, \boldsymbol{u})=\sum_{i=1}^{p} \frac{\left(\Delta_{i}^{h}(\boldsymbol{x})\right)^{2}+\left(\Delta_{i}^{v}(\boldsymbol{x})\right)^{2}+u_{i}}{2 \sqrt{u_{i}}},
$$

where $\boldsymbol{u} \in\left(R^{+}\right)^{p}$, is a $p$-dimensional vector that needs to be computed, as it will be shown below. It can be shown that $\mathrm{TV}(\boldsymbol{x}) \leq \mathrm{M}(\boldsymbol{x}, \boldsymbol{u})$ [15], and this leads to the following upper bound for $\mathcal{E}(\boldsymbol{x}, \alpha, \boldsymbol{y})$

$$
\mathcal{E}(\boldsymbol{x}, \alpha, \boldsymbol{y}) \leq\left\{\frac{1}{2 \sigma^{2}}\|\boldsymbol{x}-\boldsymbol{y}\|^{2}+\alpha \mathrm{M}(\boldsymbol{x}, \boldsymbol{u})\right\}=\mathcal{F}(\boldsymbol{x}, \boldsymbol{u}, \alpha, \boldsymbol{y}),
$$

which results in a lower bound of the integral in Eq. (18), when replacing $\mathcal{E}(\boldsymbol{x}, \alpha, \boldsymbol{y})$ by $\mathcal{F}(\boldsymbol{x}, \boldsymbol{u}, \alpha, \boldsymbol{y})$.

Then the maximization in Eq. (18) is replaced by the maximization of its lower bound, that is, we have

$$
\hat{\alpha} \approx \underset{\alpha, \boldsymbol{u}}{\operatorname{argmax}}\left\{\alpha^{\frac{p}{2}} \mathrm{p}(\alpha) \int \exp \{-\mathcal{F}(\boldsymbol{x}, \boldsymbol{u}, \alpha, \boldsymbol{y})\} \mathrm{d} \boldsymbol{x}\right\} .
$$

Note that alternatively maximizing this bound with respect to the unknowns and the auxiliary variable $\boldsymbol{u}$ results in closer bounds at each iteration. The bound in Eq. (22) is quadratic and therefore it is straightforward to evaluate analytically.

Let us examine the estimation process in Eq. (22) in detail. We can rewrite $\mathcal{F}(\boldsymbol{x}, \boldsymbol{u}, \alpha, \boldsymbol{y})$, in Eq. (21), as

$$
\begin{aligned}
\mathcal{F}(\boldsymbol{x}, \boldsymbol{u}, \alpha, \boldsymbol{y})= & \frac{1}{2}\left\{\frac{1}{\sigma^{2}} \boldsymbol{y}^{t}(\boldsymbol{y}-2 \boldsymbol{x})+\sum_{i=1}^{p} \alpha \sqrt{u_{i}}\right. \\
& \left.+\boldsymbol{x}^{t} \mathbf{Q}(\boldsymbol{u}, \alpha) \boldsymbol{x}\right\},
\end{aligned}
$$

where $t$ denotes the transpose vector, and

$$
\mathbf{Q}(\boldsymbol{u}, \alpha)=\frac{1}{\sigma^{2}} \mathbf{I}+\alpha\left(\Delta^{h^{t}} W(\boldsymbol{u}) \Delta^{h}+\Delta^{v t} W(\boldsymbol{u}) \Delta^{v}\right) .
$$

In the equations above, $\Delta^{d}$, for $d=h, v$, represents the $p \times p$ convolution matrix associated with the first order horizontal (or vertical) differences, and $W(\boldsymbol{u})$ is a $p \times p$ diagonal matrix of the form $W(\boldsymbol{u})=\operatorname{diag}\left(\boldsymbol{u}^{-\frac{1}{2}}\right)$.

Fixing $\alpha$ and $\boldsymbol{u}$ and expanding $\mathcal{F}(\boldsymbol{x}, \boldsymbol{u}, \alpha, \boldsymbol{y})$ around its minimum on $\boldsymbol{x}$ which we denote by $\overline{\boldsymbol{x}}$, we have

$$
\left.\mathcal{F}(\boldsymbol{x}, \boldsymbol{u}, \alpha, \boldsymbol{y})=\mathcal{F}(\overline{\boldsymbol{x}}, \boldsymbol{u}, \alpha, \boldsymbol{y})+(\boldsymbol{x}-\overline{\boldsymbol{x}})^{t}\right) \mathbf{Q}(\boldsymbol{u}, \alpha)(\boldsymbol{x}-\overline{\boldsymbol{x}}),
$$

and therefore

$$
\begin{aligned}
\int \exp \{-\mathcal{F}(\boldsymbol{x}, \boldsymbol{u}, \alpha, \boldsymbol{y})\} \mathrm{d} \boldsymbol{x}= & |\mathbf{Q}(\boldsymbol{u}, \alpha)|^{-\frac{1}{2}} \times \\
& \exp \{-\mathcal{F}(\overline{\boldsymbol{x}}, \boldsymbol{u}, \alpha, \boldsymbol{y})\} .
\end{aligned}
$$


Now, using Eq. (25) in Eq. (22) and differentiating the right hand side of Eq. (22) with respect to $\alpha$ and $\boldsymbol{u}$ we obtain

$$
\frac{1}{\hat{\alpha}}=\eta \frac{1}{\alpha^{o}}+(1-\eta) \frac{2}{p} \sum_{i=1}^{p} \sqrt{\hat{u}_{i}},
$$

with $\alpha^{o}=\frac{a_{\alpha}^{o}-1}{b_{\alpha}^{o}}=\operatorname{Mode}(\mathrm{p}(\alpha))$, where the hyperprior $\mathrm{p}(\alpha)$ has been defined in Eq. (13), and $\eta \in[0,1)$ is $\eta=\frac{a_{\alpha}^{o}-1}{p / 2+a_{\alpha}^{o}-1}$. In Eq. (27)

$$
\begin{aligned}
\hat{u}_{i}= & \left(\Delta_{i}^{h}(\overline{\boldsymbol{x}})\right)^{2}+\left(\Delta_{i}^{v}(\overline{\boldsymbol{x}})\right)^{2} \\
& +\operatorname{tr}\left[\mathbf{Q}(\hat{\boldsymbol{u}}, \alpha)^{-1}\left(\Delta^{h^{t}} \delta(i) \Delta^{h}+\Delta^{v t} \delta(i) \Delta^{v}\right)\right],
\end{aligned}
$$

where $\delta(i)$ is a $p \times p$ matrix with all its values equal to 0 , except $\delta(i)_{i, i}=1$. An approximation for the trace in the above equation is found by using

$$
\mathbf{Q}(\boldsymbol{u}, \alpha) \approx \overline{\mathbf{Q}}(\boldsymbol{u}, \alpha)=\frac{1}{\sigma^{2}} \mathbf{I}+\alpha z(\boldsymbol{u})\left(\Delta^{h^{t}} \Delta^{h}+\Delta^{v t} \Delta^{v}\right),
$$

with $z(\boldsymbol{u})=\frac{1}{p} \sum_{i} \frac{1}{\sqrt{u_{i}}}, \forall \boldsymbol{u} \in\left(R^{+}\right)^{p}$.

With this last approximation in mind, and substituting $\overline{\boldsymbol{x}}$ by $\hat{\boldsymbol{x}}$ defined in Eq.(19), Eq. (28) becomes

$$
\hat{u}_{i}=\left(\Delta_{i}^{h}(\hat{\boldsymbol{x}})\right)^{2}+\left(\Delta_{i}^{v}(\hat{\boldsymbol{x}})\right)^{2}+d(\hat{\boldsymbol{u}}, \hat{\alpha}),
$$

with $d(\boldsymbol{u}, \alpha)=\frac{1}{p} \operatorname{tr}\left[\overline{\mathbf{Q}}(\boldsymbol{u}, \alpha)^{-1}\left(\Delta^{h^{t}} \Delta^{h}+\Delta^{v t} \Delta^{v}\right)\right]$. Notice that the substitution of $\overline{\boldsymbol{x}}$ by $\hat{\boldsymbol{x}}$ implies that the expansion in Eq. (25) is carried out around the maximum a posteriori solution defined in Eq. (19), and that the first order term in $\hat{\boldsymbol{x}}$ is close to the one in $\overline{\boldsymbol{x}}$ which is zero. This expansion is expected to produce a better approximation to the solution of Eq. (18) than when the quadratic approximation in Eq. (22) is utilized. Notice also that $\hat{\boldsymbol{x}}$ can be easily calculated using Cambolle's algorithm.

Finally the proposed iterative method is summarized in Algorithm 1.

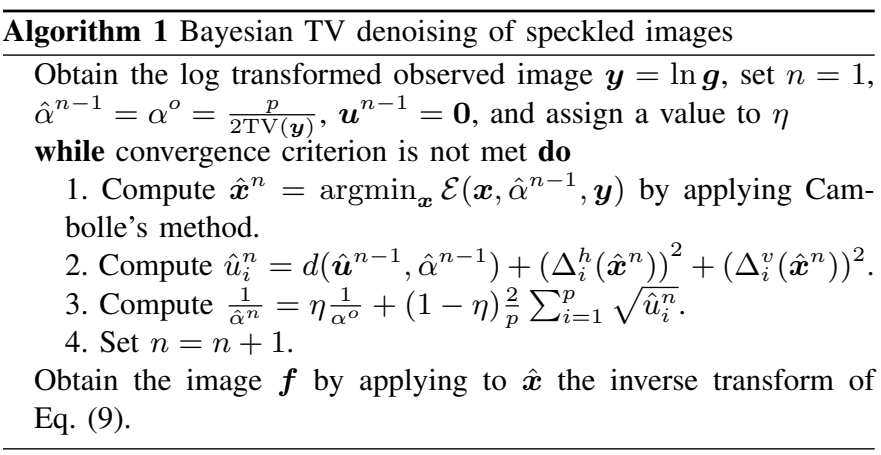

\section{EXPERIMENTAL RESULTS}

A number of experiments have been carried out in order to evaluate the performance of the proposed algorithm 1 (henceforth referred to as LTV), compare with the method in [2], and with a Wiener restoration of the $\boldsymbol{y}$ image, defined in Eq. (6), to which the inverse transform of Eq. (9) is later applied to obtain $\hat{\boldsymbol{f}}_{W i e}$ (henceforth referred to as $L W i e$ ).

The proposed algorithm was ran until the criterion $\| \hat{\alpha}^{k}-$ $\hat{\alpha}^{k-1} \| / \max \left(\hat{\alpha}^{k}, \hat{\alpha}^{k-1}\right)<10^{-3}$ was satisfied, or a number of 10 iterations reached, with $\hat{\alpha}^{k}$ denoting the $\hat{\alpha}$ estimate at the $k$ iteration step. We have used for $\eta$, defined in Eq. (27), the value $\eta=1-0.8 / L$, which prevents an experimentally observed trend, of the proposed

\begin{tabular}{|c|c|c|c|c|c|c|c|}
\hline & \multicolumn{2}{|c|}{ [2] } & \multicolumn{2}{|c|}{ LWie } & \multicolumn{2}{|c|}{ LTV } & \\
\hline Image & PSNR & SSIM & PSNR & SSIM & PSNR & SSIM & $\mathrm{L}$ \\
\hline \multirow{4}{*}{ Fig. 1(a) } & 23.11 & 0.4922 & 14.05 & 0.1777 & 23.45 & 0.5477 & 4 \\
\hline & 26.60 & 0.6694 & 20.89 & 0.4494 & 26.90 & 0.6909 & 16 \\
\hline & 27.54 & 0.7169 & 24.00 & 0.6028 & 28.28 & 0.7425 & 32 \\
\hline & 28.15 & 0.7460 & 27.07 & 0.7404 & 29.95 & 0.8014 & 64 \\
\hline \multirow{4}{*}{ Fig. 1(f) } & 17.69 & 0.3336 & 9.67 & 0.1172 & 18.32 & 0.4192 & 4 \\
\hline & 22.63 & 0.5486 & 15.34 & 0.2794 & 23.53 & 0.6437 & 16 \\
\hline & 24.29 & 0.6465 & 18.05 & 0.3818 & 25.27 & 0.7259 & 32 \\
\hline & 25.47 & 0.7251 & 20.91 & 0.4941 & 27.03 & 0.7954 & 64 \\
\hline \multirow{4}{*}{ Lena } & 20.97 & 0.3616 & 11.34 & 0.1019 & 22.45 & 0.5203 & 4 \\
\hline & 26.42 & 0.5785 & 17.71 & 0.2549 & 28.58 & 0.7638 & 16 \\
\hline & 28.68 & 0.6798 & 20.71 & 0.3542 & 30.53 & 0.8207 & 32 \\
\hline & 30.50 & 0.7638 & 23.70 & 0.4649 & 32.27 & 0.8610 & 64 \\
\hline \multirow{4}{*}{ camera } & 20.08 & 0.4186 & 11.10 & 0.2432 & 20.94 & 0.5173 & 4 \\
\hline & 23.48 & 0.5563 & 17.40 & 0.4015 & 26.01 & 0.7214 & 16 \\
\hline & 24.57 & 0.6380 & 20.53 & 0.4790 & 28.03 & 0.7912 & 32 \\
\hline & 25.21 & 0.7096 & 23.60 & 0.5569 & 29.99 & 0.8429 & 64 \\
\hline
\end{tabular}

TABLE I

VALUES OF PSNR AND SSIM FOR DIFFERENT RESTORATIONS OF THE SYNTHETIC IMAGE SET, FOR DIFFERENT NUMBER OF LOOKS $L$ MULTIPLICATIVE NOISE.

algorithm 1 , to obtain too high values for $\alpha$ when the value of the number of looks $L$ grows, producing an extra-smoothing effect.

We first deal with synthetic images. Two aerial images, a $1024 \times$ 1024 image, a detail of which is shown in Fig. 1(a), and a $512 \times 512$ one, a detail of which is shown in Fig. 1(f). The $512 \times 512$ Lena and the $256 \times 256$ cameraman images have also been used in the experiments. Multiplicative noise, corresponding to different number of looks $L$, has been generated and the resulting degraded images were restored applying the various methods. Table I shows a numerical comparison of the different methods both in term of the peak signalto-noise ratio (PSNR), and the Structural Similarity Index Measure (SSIM) defined in [16], whose maximal value, corresponding to exactly equal images, is +1 . In Fig. 1 details from a $360 \times 360$ segment of the original, degraded, and the different restorations, for the two aerial images are shown. Both the numerical and the visual comparison of the restored images support the better performance of the proposed algorithm.

Finally a real TerraSAR-X Enhanced Ellipsoid Corrected (EEC) $\mathrm{HH}$ polarization channel image, from near Rome, has been considered. A $256 \times 256$ image zone of a 25 looks observation is shown in Fig. 2(a), and its corresponding restoration using the proposed method is shown in Fig. 2(b). In this case the real noise free image is not available in order to perform a numerical evaluation of the restoration quality, but the reduction of speckle in the restored image is visually appreciable.

\section{CONCLUSions}

A novel algorithm for despeckling images, within the logtransformed spatial domain, using a TV prior whose model parameter is automatically determined using Evidence Analysis within the Hierarchical Bayesian Paradigm, has been proposed in this paper. Both the numerical and the visual evaluation of the quality of the restored images support the effectiveness of the proposed algorithm.

\section{REFERENCES}

[1] C. Oliver and S. Quegan, Understanding Synthetic Aperture Radar Images. Raleigh, NC: SciTech Publishing, Inc, 2004.

[2] J.-S. Lee, "Refined filtering of image noise using local statistics," Comput. Graph. Image Process., vol. 15, no. 1, pp. 380-389, 1981.

[3] A. Lopes, R. Touzit, and E. Nezry, "Adaptive speckle filters and scene heterogeneity," IEEE Trans. Geosci. Remote Sens., vol. 28, no. 6, pp. 992-1000, 1990. 


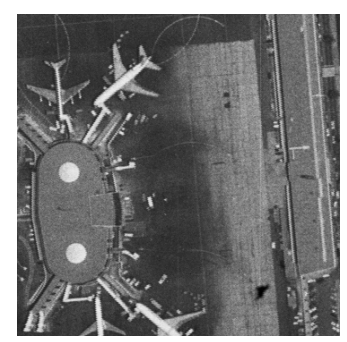

a

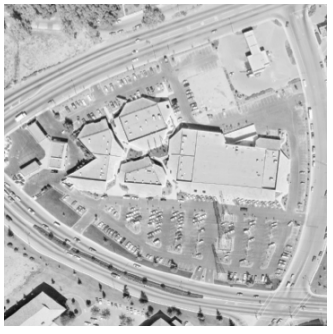

$\mathrm{f}$

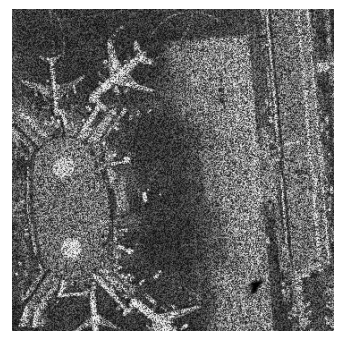

b

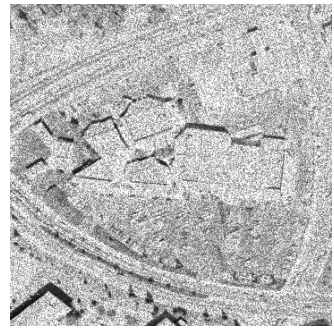

$\mathrm{g}$

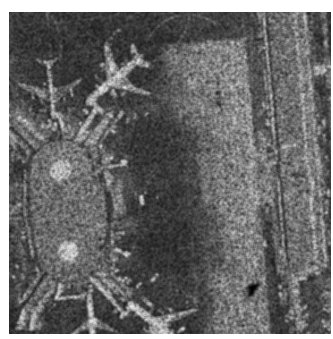

c

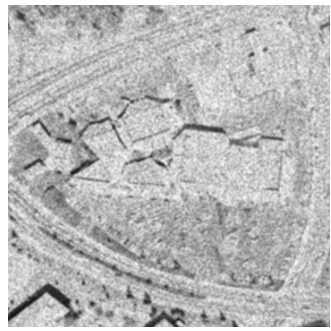

h

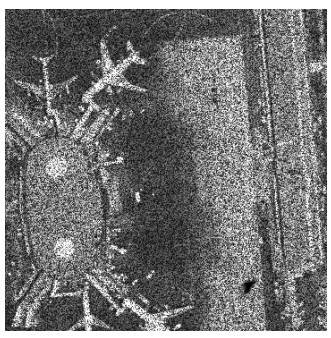

d

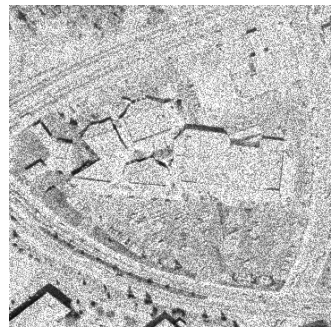

i

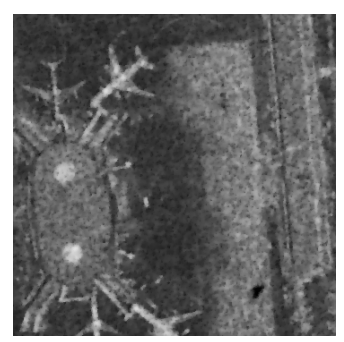

e

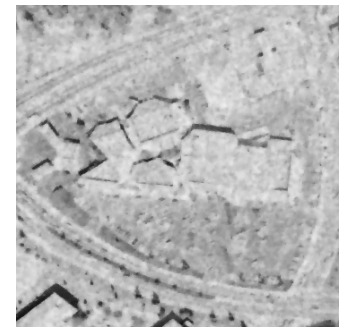

Fig. 1. $360 \times 360$ details of: (a) \& (f) aerial original images, their corresponding degradations for (b) 4 looks and (g) 16 looks, (c) \& (h) their restorations using the method in [2], (d) \& (i) using LWie and (e) \& (j) using the proposed LTV method.

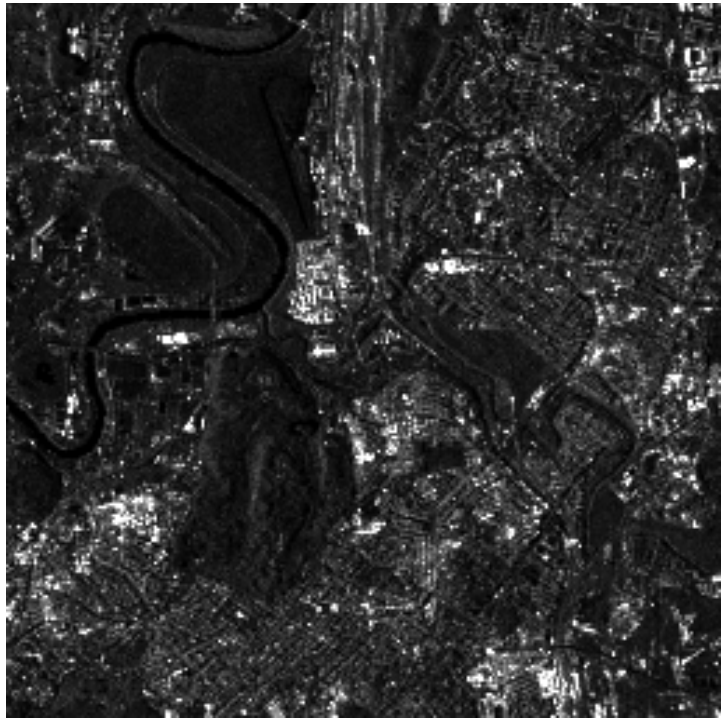

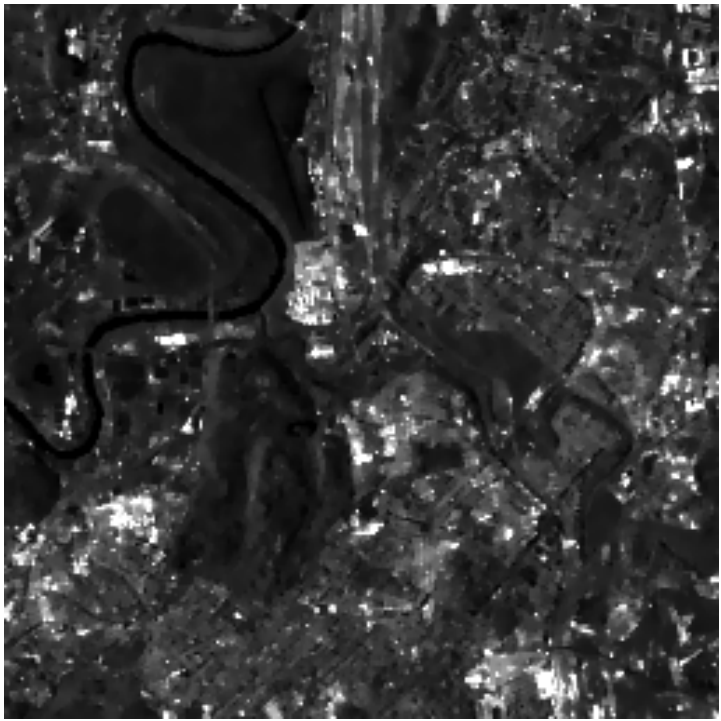

b

Fig. 2. (a) $256 \times 256$ detail of a EEC HH TerraSAR-X image, and (b) its restoration using the proposed LTV method.

[4] J.-H. Hou, "Speckle reduction algorithm for synthetic aperture radar images based on bayesian maximum a posteriori estimation in wavelet domain," Opt. Eng., vol. 47, no. 5, pp. 057004-1-11, 2008.

[5] M. Amirmazlaghani and H. Amindavar, "Two novel bayesian multiscale approaches for speckle suppression in sar images," IEEE Trans. Geosci. Remote Sens., vol. 48, no. 7, pp. 2980-2993, 2010.

[6] S. Durand, J. Fadili, and M. Nikolova, "Multiplicative noise removal using 11 fidelity on frame coefficients," Jour. of Math. Imaging and Vision, vol. 36, no. 3, pp. 201-226, 2010.

[7] G. Aubert and J.-F. Aujol, "A variational approach to removing multiplicative noise," SIAM Journal on Applied Mathematics, vol. 68, no. 4, pp. 925-946, 2008. [Online]. Available: http://link.aip.org/link/?SMM/68/925/1

[8] J. Bioucas-Dias and M. Figueiredo, "Total variation restoration of speckled images using a split-bregman algorithm," in Image Processing (ICIP), 2009 16th IEEE International Conference on, Nov 2009, pp. 3717-3720.

[9] L.-L. Huang, L. Xiao, and Zhi-HuiWei, "Multiplicative noise removal via a novel variational model," EURASIP Jour. on Image and Video Proc., vol. 2010, no. ID 250768, pp. 1-16, 2010.
[10] R. Molina, A. K. Katsaggelos, and J. Mateos, "Bayesian and regularization methods for hyperparameter estimation in image restoration," IEEE Trans. Image Process., vol. 8, no. 2, pp. 231-246, 1999.

[11] H. Xie, L. E. Pierce, and F. T. Ulaby, "Statistical properties of logarithmically transformed speckle," IEEE Trans. Geosci. Remote Sens. vol. 40, no. 3, pp. 721-727, 2002.

[12] J.-L. Starck, F. Murtagh, and J. M. Fadili, Sparse Image and Signal Processing. Cambridge University Press, 2010.

[13] A. Chambolle, "An algorithm for total variation minimization and applications," Jour. of Math. Imaging and Vision, vol. 20, pp. 89-97, 2004.

[14] L. I. Rudin, S. Osher, and E. Fatemi, "Nonlinear total variation based noise removal algorithms," Physica D, pp. 259-268, 1992.

[15] S. D. Babacan, R. Molina, and A. Katsaggelos, "Parameter estimation in TV image restoration using variational distribution approximation," IEEE Trans. Image Process., no. 3, pp. 326-339, March 2008.

[16] Z. Wang, A. C. Bovik, H. R. Sheikh, and E. P. Simoncelli, "Image quality assessment: From error measurement to structural similarity," IEEE Trans. on Img Proc., vol. 13, no. 4, pp. 600-612, 2004. 